\title{
Characteristics of patients who made a return visit within 72 hours to the emergency department of a Singapore tertiary hospital
}

Amy Hui Sian $\underline{\mathrm{Chan}}^{1}$, MPH, Shu Fang $\underline{\mathrm{Ho}}^{2}$, BAcc, Stephanie Man Chung Fook-Chong ${ }^{3}$, MSc, Sherman Wei Qiang $\underline{\text { Lian }}^{4}$, Dip(Nursing), Nan Liu ${ }^{4,5}$, PhD, Marcus Eng Hock $\underline{\text { Ong }}^{4,6}$, MBBS, MPH

\begin{abstract}
INTRODUCTION 72-hour emergency department (ED) reattendance is a widely-used quality indicator for quality of care and patient safety. It is generally assumed that patients who return within 72 hours of ED discharge (72-hour re-attendees) received inadequate treatment or evaluation. The current literature also suggests considerable variation in probable causes of 72-hour ED reattendances internationally. This study aimed to understand the characteristics of these patients at the ED of a Singapore tertiary hospital.

METHODS We conducted a retrospective cohort study on all ED visits between 1 January 2013 and 31 December 2013. 72-hour re-attendees were compared against non-re-attendees based on patient demographics, mode of arrival, patient acuity category status (i.e. P1/P2/P3/P4), seniority ranking of doctor-in-charge and medical diagnoses. Multivariate analysis using the generalised linear model was conducted on variables associated with 72-hour ED re-attendance.

RESULTS Among 104,751 unique patients, 3,065 (2.93\%) were in the 72-hour re-attendees group. Multivariate analysis showed that the following risk factors were associated with higher risk of returning within 72 hours: male gender, older age, arrival by ambulance, triaged as $\mathrm{P} 2$, diagnoses of heart problems, abdominal pain or viral infection (all $p<0.001$ ), and Chinese ethnicity $(p=0.006)$. There was no significant difference in the seniority ranking of the doctor-in-charge between both groups $(p=0.419)$.

CONCLUSION Several patient and event factors were associated with higher risk of being a 72-hour re-attendee. This study forms the basis for hypothesis generation and further studies to explore reasons behind reattendances so that interventions can be developed to target high-risk groups.
\end{abstract}

Keywords: 72-hour emergency department reattendance, 72-hour emergency department return, emergency department reattendance, emergency department return

\section{INTRODUCTION}

72-hour emergency department (ED) reattendance is a widelyused quality indicator for quality of care and patient safety. Patients who return within 72 hours of discharge from the ED are often perceived to have received inadequate treatment or evaluation. ${ }^{(1)}$ In a review, about $9 \%-48 \%$ of all readmissions were judged to be preventable, as they were associated with indicators of substandard care during the index hospitalisation. ${ }^{(1)}$

Reasons behind 72-hour ED reattendance can be classified into three broad categories, namely illness-, doctor- and patient-related ED returns. ${ }^{(2)}$ In illness-related ED returns, the patient receives appropriate emergency medical care, but disease progression prompts the return. In doctor-related ED returns, the patient returns due to the doctor's (mis)behaviour. Unscheduled returns were found to be associated with medical errors in prognosis, treatment, followup care and information. ${ }^{(3)}$ In patient-related ED returns, there is no evidence of lapses in medical care and returns are primarily patientinitiated. However, it can be difficult to differentiate between the natural course of a disease, suboptimal therapy, overanxious patient reaction and medical error as the cause of the return. ${ }^{(4)}$

Reattendance within 72 hours of the patient's discharge from the ED may contribute to an unnecessary increase in ED patient load. It may also suggest inadequate patient assessment, treatment or even discharge care instructions. ${ }^{(5,6)}$ While rates above $5 \%$ may reflect poor-quality care, rates below $1 \%$ reflect excessive risk aversion. ${ }^{(7)}$ This implies a need for a more in-depth analysis of the causes of unscheduled 72-hour ED reattendance for policymakers and hospital administrators.

In Singapore, 72-hour ED reattendance is a key performance indicator for most EDs. A report published by Changi General Hospital, Singapore, in 2014 quoted a national reattendance rate of $4.3 \% .{ }^{(5)}$ Hence, for every $23 \mathrm{ED}$ attendances, one was a 72-hour ED reattendance. Taking the average of 400 cases per day, about 17 patients revisited the ED per day within 72 hours of discharge. However, the causes of these 72-hour ED reattendances in Singapore are not well understood, as the published data that characterises such patients is limited. Therefore, understanding the patient characteristics associated with 72-hour ED reattendances in Singapore could help to identify factors associated with it; appropriate interventions can subsequently be undertaken to reduce the rates of preventable reattendances.

In this data mining analysis of electronic health records, we aimed to analyse the characteristics of patients who returned

\footnotetext{
${ }^{1}$ Saw Swee Hock School of Public Health, National University of Singapore, ${ }^{2}$ Duke-NUS Graduate Medical School, ${ }^{3}$ Division of Research, ${ }^{4}$ Department of Emergency Medicine, Singapore General Hospital, ${ }^{5}$ Centre for Quantitative Medicine, ${ }^{6} \mathrm{Health}$ Services and Systems Research, Duke-NUS Graduate Medical School, Singapore

Correspondence: Ms Ho Shu Fang, MD Candidate, Singapore General Hospital, Department of Emergency Medicine, Outram Road, Block 1 Level 3, DEM Office C, Singapore 169608.shufang@u.duke.nus.edu
} 
within 72 hours of discharge from ED, comparing them to those who did not return within 72 hours, at the ED of Singapore General Hospital (SGH), Singapore. We also aimed to identify possible factors that contributed to 72 -hour ED reattendance. This would help us to understand the profile of 72-hour ED re-attendees, as well as create a scientific basis to generate hypotheses for future related studies.

\section{METHODS}

SGH is one of Singapore's largest restructured acute tertiary hospitals, accounting for about one-third of total acute hospital beds in the public sector and about a quarter of acute beds nationwide. Annually, the ED sees about 140,000 patients, with $300-500$ visits per day. The inpatient wards admit an average of 89,000 patients annually, an average of 240 admissions per day.

This was a retrospective cohort study analysing variables extracted from the electronic health intelligence system (eHINTS) at the SGH ED, using Oracle Business Intelligence Enterprise Edition software. eHINTS consolidates and analyses patient and healthcare data from hospital electronic health records that are uploaded on the web-based business intelligence platform. The information is easily accessed online by the hospital's doctors and healthcare staff. ${ }^{(8)}$ The study population included patients who visited the SGH ED between 1 January 2013 and 31 December 2013. All patients who were aged 21 years and above, and had visited the SGH ED during the study period were included. We excluded patients who left without being seen (LWBS), were discharged at their own risk (AOR), absconded (ABS) or were dead on arrival (DOA). As this was a retrospective data-mining cohort study, variables were chosen based on their availability and reliability in eHINTS. In practice, this meant that we only analysed structured data in eHINTS and left out unstructured (free text) data. The study was approved by the SingHealth Centralised Institutional Review Board.

The included patients were divided into two groups for comparison: those who returned within 72 hours of discharge from ED (72-hour re-attendees group) and those who did not return within 72 hours (non-re-attendees group). The reattendance window was defined as the time from the index visit discharge to registration at the second visit. The index visit for the 72-hour re-attendees group was defined as the first of two visits made by a patient who returned within 72 hours of discharge from the ED. The index visit for the non-re-attendees group was defined as the first visit made by the patient within the study period. We compared and analysed the following patient demographics and variables associated with the index visit: gender, age, ethnicity, mode of arrival, patient acuity category (PAC) status (i.e. P1/P2/P3/ $\mathrm{P} 4)$, seniority ranking of doctor-in-charge and medical diagnosis.

At $\mathrm{SGH}^{\prime}$ s ED, trained registered nurses assess a patient's presenting medical condition and assign a specific priority level based on the patient's presenting condition using the PAC triage system. There are four levels of ED priority: P1 for patients who are critically ill, P2 for non-ambulant patients with major emergencies, P3 for ambulant patients with minor emergencies and P4 for non-emergency cases. In addition, the patient's medical diagnosis is coded according to the International Classification of Diseases, Ninth Revision (ICD-9). The ICD-9 is designed to promote international comparability in the collection, processing, classification and presentation of mortality statistics. ${ }^{(9)}$ For the purposes of this study, we recategorised the ICD-9 diagnoses into broader categories by engaging a computer engineering intern to filter and group the ICD-9 diagnoses with reference to ICD coding information.

Data analysis was performed using STATA version 13 (StataCorp, College Station, TX, USA). Two patient groups were compared by first identifying the variables that were significantly different using analysis of two-way tables (measures of association). Multivariate analysis using the generalised linear model (relative risk [RR]) was then conducted on variables associated with 72-hour ED reattendance. RR, also known as risk ratio, was reported, as this was a retrospective cohort study and RR was more appropriate for our study design compared to odds ratios. The level of statistical significance was set at $\mathrm{p}<0.05$.

\section{RESULTS}

A total of 104,751 unique patients were seen between 1 January 2013 and 31 December 2013. 10,401 (9.93\%) LWBS, AOR, ABS and DOA patients were excluded from the study. Of the remaining 94,350 eligible patients, 3,065 (3.25\%) were in the 72 -hour re-attendees group and 91,285 (96.75\%) in the non-re-attendees group (Fig. 1). The demographic characteristics of both groups are shown in Table I. There were statistically significant differences between the two groups in relation to gender $(p<0.001)$, age $(p<0.001)$ and ethnicity $(p<0.05)$. Ethnicity was analysed based on four broad categories: Chinese, Indian, Malays and Others.

Table II shows the variables associated with the index visit of both groups. Univariate analysis revealed significant differences between the two groups of patients in relation to their mode of arrival $(p=0.005)$, PAC $(p<0.001)$ and medical diagnosis $(p<0.001)$. There were no significant differences between the two groups in relation to the seniority of the doctor-in-charge $(p=0.419)$. The seniority ranking of the doctor-in-charge was

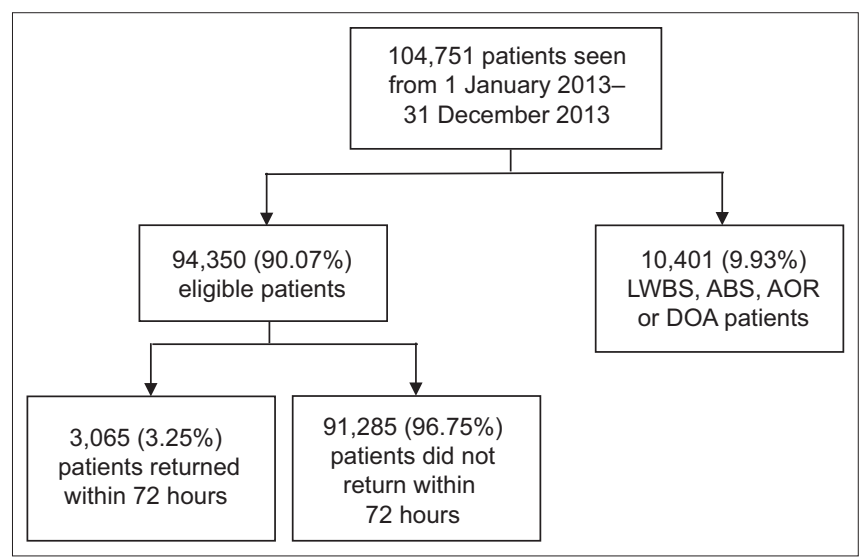

Fig. 1 Flowchart shows the study selection process. ABS: absconded; AOR: discharged at own risk; DOA: dead on arrival; LWBS: left without being seen 
analysed based on whether the doctor was junior or senior; junior doctors were non-specialists (including specialist trainees) and senior doctors were specialists. The top 11 ICD-9 diagnoses

Table I. Demographics of patients included in the study $(n=94,350)$.

\begin{tabular}{lccc}
\hline Variable & \multicolumn{2}{c}{ No. (\%) } & $\begin{array}{c}\text { Stratum-specific } \\
\text { p-value }\end{array}$ \\
\cline { 2 - 3 } & $\begin{array}{c}\text { 72-hr } \\
\text { re-attendees } \\
(\mathbf{n}=\mathbf{3 , 0 6 5 )}\end{array}$ & $\begin{array}{c}\text { No-attendees } \\
\text { (n= 91,285) }\end{array}$ & \\
\hline Gender & & & \\
Male & $1,372(44.76)$ & $44,648(48.91)$ & \\
Female & $1,693(55.24)$ & $46,637(51.09)$ & \\
Age group (yr) & & & \\
21-30 & $574(18.73)$ & $18,999(20.81)$ & \\
31-40 & $569(18.56)$ & $14,662(16.06)$ & \\
41-50 & $487(15.89)$ & $13,276(14.54)$ & \\
51-60 & $581(18.96)$ & $15,358(16.82)$ & \\
61-70 & $399(13.02)$ & $13,264(14.53)$ & \\
71-80 & $299(9.76)$ & $10,132(11.10)$ & \\
$\geq 81$ & $156(5.09)$ & $5,594(6.13)$ & \\
Ethnicity & & & \\
Chinese & $2,001(65.29)$ & $58,805(64.42)$ & \\
Indian & $400(13.05)$ & $11,195(12.26)$ & \\
Malay & $312(10.18)$ & $10,768(11.80)$ & \\
Others & $352(11.48)$ & $10,517(11.52)$ & \\
\hline
\end{tabular}

during the study period were found to be general symptoms, diseases of the heart, respiratory infections, intestinal infections, abdominal pain, ear conditions, spondylosis/intervertebral disc disorders/other back problems, gastritis and duodenitis, viral infections, eye disorders and others.

Multivariate analysis showed that some factors were associated with a higher risk of returning within 72 hours (Table III). In terms of demographic variables, female patients had a relatively lower risk (RR 0.88; $p<0.001$ ) of returning within 72 hours than male patients. The age group $\geq 81$ years had a relatively higher risk (RR 1.59; $\mathrm{p}<0.001$ ) compared to those aged $21-30$ years. Malay patients had a relatively lower risk (RR 0.84; $p=0.006$ ) than Chinese patients. Regarding variables related to the index ED visit, patients who did not arrive by ambulance had a relatively lower risk (RR 0.77; $p<0.001$ ) of returning within 72 hours than patients who arrived by ambulance. Patients triaged as $\mathrm{P} 2 \mathrm{had}$ a relatively higher risk (RR 2.69; $p<0.001)$ than patients triaged as P3/P4. Patients diagnosed with diseases of the heart (RR 3.26; $p<0.001$ ), viral infection (RR 2.80; $p<0.001$ ) and abdominal pain (RR 2.15; $p<0.001)$ had a relatively higher risk of returning within 72 hours than those diagnosed with general symptoms.

\section{DISCUSSION}

From the multivariate analysis, factors associated with higher risk for 72-hour ED reattendance included male gender, advanced

Table II. Patients' mode of arrival and doctor's ranking, patient acuity category status and top 11 International Classification of Diseases, Ninth Revision (ICD-9) diagnoses, by study group $(n=94,350)$.

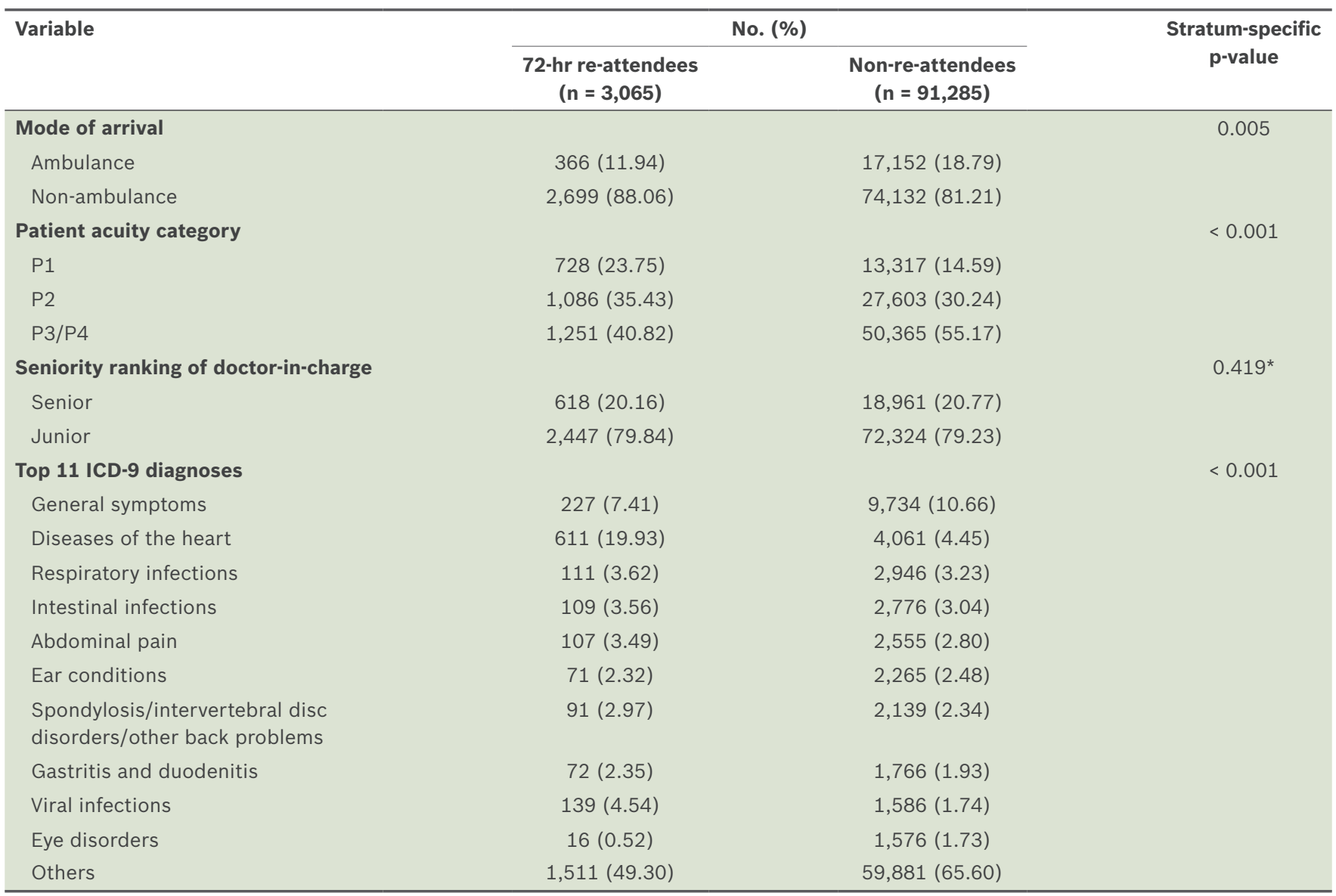

$*_{p}$-value is not significant. 
Table III. Multivariate analysis with generalised linear model (relative risk [RR]) of variables associated with 72-hour emergency department reattendance.

\begin{tabular}{|c|c|c|}
\hline Variable & Adjusted RR (95\% CI) & p-value \\
\hline \multicolumn{3}{|l|}{ Gender } \\
\hline Male & 1.00 & ref \\
\hline Female & $0.88(0.82,0.95)$ & $<0.001$ \\
\hline \multicolumn{3}{|l|}{ Age group (yr) } \\
\hline $21-30$ & 1.00 & ref \\
\hline $31-40$ & $1.24(1.11,1.39)$ & $<0.001$ \\
\hline $41-50$ & $1.18(1.05,1.33)$ & 0.007 \\
\hline $51-60$ & $1.31(1.17,1.48)$ & $<0.001$ \\
\hline $61-70$ & $1.20(1.06,1.37)$ & 0.005 \\
\hline $71-80$ & $1.41(1.22,1.62)$ & $<0.001$ \\
\hline$\geq 81$ & $1.59(1.33,1.90)$ & $<0.001$ \\
\hline \multicolumn{3}{|l|}{ Ethnicity } \\
\hline Chinese & 1.00 & ref \\
\hline Indian & $0.96(0.87,1.07)$ & $0.511^{*}$ \\
\hline Malay & $0.84(0.76,0.96)$ & 0.006 \\
\hline Others & $0.90(0.81,1.02)$ & $0.098^{*}$ \\
\hline \multicolumn{3}{|l|}{ Mode of arrival } \\
\hline Ambulance & 1.00 & ref \\
\hline Non-ambulance & $0.77(0.69,0.86)$ & $<0.001$ \\
\hline \multicolumn{3}{|l|}{ Patient acuity category } \\
\hline P3/P4 & 1.00 & ref \\
\hline $\mathrm{P} 1$ & $2.07(1.89,2.26)$ & $<0.001$ \\
\hline $\mathrm{P} 2$ & $2.69(2.40,3.03)$ & $<0.001$ \\
\hline \multicolumn{3}{|l|}{ Top 11 ICD-9 diagnoses } \\
\hline General symptoms & 1.00 & ref \\
\hline Diseases of the heart & $3.26(2.77,3.84)$ & $<0.001$ \\
\hline Respiratory infections & $1.05(0.81,1.35)$ & $0.721^{*}$ \\
\hline Intestinal infections & $1.43(1.14,1.80)$ & 0.002 \\
\hline Abdominal pain & $2.15(1.72,2.69)$ & $<0.001$ \\
\hline Ear conditions & $1.03(0.79,1.34)$ & $0.818^{*}$ \\
\hline $\begin{array}{l}\text { Spondylosis/intervertebral } \\
\text { disc disorders/other back } \\
\text { problems }\end{array}$ & $1.52(1.20,1.93)$ & 0.001 \\
\hline Gastritis and duodenitis & $1.38(1.06,1.78)$ & 0.016 \\
\hline Viral infections & $2.80(2.21,3.54)$ & $<0.001$ \\
\hline Eye disorders & $0.40(0.24,0.66)$ & $<0.001$ \\
\hline Others & $1.09(0.95,1.25)$ & $0.229 *$ \\
\hline
\end{tabular}

* $\mathrm{p}$-value is not significant. $\mathrm{Cl}$ : confidence interval; ICD-9: International Classification of Diseases, Ninth Revision; ref: reference group

age, arrival by ambulance, P2 PAC status, diagnoses of heart problems, abdominal pain or viral infections (all $p<0.001$ ), and Chinese ethnicity $(p=0.006)$. Although the univariate analysis showed more female than male patients in the 72-hour re-attendees group, in the multivariate analysis, male gender was associated with a higher risk of reattendance. The likely explanation for the discrepancy between the univariate and multivariate results was that the elderly patient group had a greater proportion of female patients, as women live longer than men. After adjusting for age and other confounders in the multivariate analysis, male patients were found to be at relatively higher risk of returning within 72 hours. Likewise, although the univariate analysis showed a greater portion of younger patients compared to older patients in the 72-hour re-attendees group, the multivariate analysis showed that older age was associated with a higher risk of reattendance. As older patients are known to have atypical or very non-specific geriatric syndromes (e.g. delirium, dizziness, syncope, falling, weight loss and incontinence), it is possible that patients in the elderly group were discharged without being adequately treated in the index visit, resulting in the need to return.

We found that 72-hour re-attendees tended to present with a higher PAC status of P2 and above, which could suggest that these patients were sicker. However, our analysis could not determine if their revisit was related to the preceding visit or the patient was of $\mathrm{P} 2$ and above status; the latter could suggest inadequate treatment on their first visit. The results showed that P1 patients had a higher relative risk of returning (RR 2.07; $p<0.001)$ than P3 patients. It has been hypothesised that there are significant differences in the medical diagnoses of 72-hour re-attendees and non-re-attendees; this is because certain diagnoses can potentially indicate a more severe disease process and outcome. ${ }^{(9)} \mathrm{A}$ literature review showed that similar previous studies analysed patient diagnoses and chief complaints in broad categories such as general symptoms; psychiatric; cardiovascular; ear, nose and throat; pulmonary; gastrointestinal; and dermatological conditions. ${ }^{(2,4,10,11)}$

Having identified the three most common diagnoses that were the greatest risk factors in the 72-hour re-attendees group, namely heart problems, viral infection and abdominal pain, we observed changes in the most common presenting diagnosis in Singapore over the last decade. In 1994, a retrospective study $(n=166)$ conducted at the ED of Toa Payoh Hospital, Singapore, found the most common returning diagnosis to be asthma. ${ }^{(12)}$ It also found that more than two-thirds of patients return to the ED due to a failure to improve from their initial condition. In 2005, a retrospective study $(n=842)$ at the ED of National University Hospital, Singapore, found that 25\% of 72-hour re-attendees presented with abdominal pain and more than half of them were then admitted. ${ }^{(13)}$ In the present study, however, patients with heart problems were the most likely to return to the ED, although the underlying cause of the revisits was unclear. In addition, the 'diseases of the heart' diagnosis included a broad spectrum of pathology involving the conduction system, coronary arteries, myocardium and valves. The disease process and treatment for each pathology also varied greatly.

In future studies, we aim to look beyond the index visit and investigate if there was a significant difference in diagnosis and disposition status between the index and return ED visit. It would also be useful to examine admission outcomes of the revisit in the subgroup of 72-hour re-attendees who were admitted on their return visit, particularly mortality and complication rates, as research has established that patients who are admitted on the return visit have poor health outcomes. ${ }^{(14)}$ 
Lastly, it would be interesting to study the reasons why patients who arrived by ambulance were more likely to return. This could be an indication of inadequate medical treatment on the first visit and improper usage of medical services by overanxious patients. Based on our clinical experience, some 72-hour ED reattendances by ambulance resulted from ambulance activation by nursing home staff. The nursing home could have had inadequate resources to cope with the patient's symptoms and hence sent the patient back to the hospital shortly after ED discharge.

The present study had some limitations. To achieve this major cohort study with a large population sample $(n=94,350)$, a retrospective data mining approach was used, where only structured variables were collected from the eHINTS database and analysed. We did not analyse unstructured data fields (free text) or review the text of the clinical notes. As this was an unfunded study with resource constraints, unique patients were studied, instead of unique ED visits. Hence, 72-hour re-attendees who reattended more than once in the study period were not accounted for.

We speculated that the group of 72-hour re-attendees who had viral infection returned due to inadequate patient education or discharge instructions; patients may have felt that their persistent fever was not improving and wanted another medical review, while others may have visited to renew their medical leave. However, further research is required to ascertain the reasons why patients return within 72 hours of discharge from the ED. This information has to be extracted from other sources such as patients' clinical notes. It might be fruitful to do a qualitative study from the patient's perspective to explore the reasons for a return ED visit. Rising et al ${ }^{(15)}$ found that a patient's perceived inability to access timely follow-up care and uncertainty about disease progression were primary motivators for 72-hour ED reattendance. Many patients also preferred hospital-based care because of increased convenience and timely results. ${ }^{(15}$ Nevertheless, differentiation between the natural course of a disease, suboptimal therapy, overanxious reaction of the patient and medical errors remains difficult. ${ }^{(4)}$ A literature review on 72-hour ED reattendances revealed that there were considerable variations in the probable causes $^{(1-4,6,10,11,15-19)}$ and that they remained ill-defined. ${ }^{(20)}$

In addition, repeated ED attendances due to patient-related reasons (e.g. free medical consultation card, psychiatric issues and social issues) are known to be poor indicators for the quality of care rendered. ${ }^{(10,14)}$ In the Hong Kong study, it was found that patients who return for such reasons were responsible for about $10 \%$ of unscheduled return visits. ${ }^{(2)}$ Due to the dataset limitation, this patient group was not excluded in the study; therefore, we were unable to extrapolate that inadequate medical care in the preceding visit was a reason for 72-hour ED reattendance. We were also unable to identify cases that were recalled by the department for review due to abnormal results.

As the data collection and analysis were performed using SGH's ED database, eHINTS, based on the hospital's patient profile, the results collected in this study might not be generalisable to other healthcare institutions in Singapore. There was also no proof that the non-re-attendees did not seek medical treatment at other EDs in Singapore.

For future research, it would be useful to look at the time of presentation variable. A study by Goldman et $\mathrm{al}^{(6)}$ found that paediatric patients seen during the ED's busiest hours were more likely to return within 72 hours. A further analysis focusing on the day of the week and time of the day, to determine if these variables affect reattendance rates, would provide useful information for manpower roster planning in the ED. Our finding that the seniority ranking of the attending doctor had no significant effect on the 72-hour ED reattendance rate would also be useful in this area. Another resource-related confounder (i.e. bed-occupancy rate of the hospital) has been shown to affect the 72-hour ED reattendance rate ${ }^{(21)}$ and should be included in future studies.

In conclusion, we found that several patient and event factors were associated with higher risk of being a 72-hour re-attendee. The risk factors include male gender, Chinese ethnicity, advanced age, arrival by ambulance, P2 acuity, and diagnoses of heart problems, abdominal pain or viral infections. This study forms the basis for hypothesis generation and further studies to explore reasons behind reattendances so that interventions can be developed to target high-risk groups.

\section{REFERENCES}

1. Benbassat J, Taragin M. Hospital readmissions as a measure of quality of health care: advantages and limitations. Arch Intern Med 2000; 160:1074-81.

2. $\mathrm{Ng} \mathrm{CP}$, Chung $\mathrm{CH}$. An analysis of unscheduled return visits to the accident and emergency department of a general public hospital. Hong Kong J Emerg Med 2003; 10:153-61.

3. Nuñez S, Hexdall A, Aguirre-Jaime A. Unscheduled returns to the emergency department: an outcome of medical errors? Qual Saf Health Care 2006; 15:102-8.

4. Wu CL, Wang FT, Chiang YC, et al. Unplanned emergency department revisits within 72 hours to a secondary teaching referral hospital in Taiwan. J Emerg Med 2010; 38:512-7.

5. Emergency Department and Quality Management Office, Changi General Hospital, Singapore. Re-Attendance at Emergency Department. In: Changi General Hospital, Singapore [online]. Available at: http://www.cgh.com.sg/ Medical_Specialities/Clincal\%20Outcomes/Pages/reattendance_outcome. aspx. Accessed May 13, 2016.

6. Goldman RD, Ong M, Macpherson A. Unscheduled return visits to the pediatric emergency department-one-year experience. Pediatr Emerg Care 2006; 22:545-9.

7. Heyworth J. Emergency medicine-quality indicators: the United Kingdom perspective. Acad Emerg Med 2011; 18:1239-41.

8. Integrated Health Information Systems. Project Showcase: Electronic Health Intelligence System (eHINTS). Available at: http://www.ihis.com. sg/services/Pages/eHINTS.aspx. Accessed May 13, 2016.

9. Centers for Disease Control and Prevention. International Classification of Diseases, Ninth Revision (ICD-9). Available at: http://www.cdc.gov/nchs/ icd/icd9.htm. Accessed May 13, 2016.

10. Pham JC, Kirsch TD, Hill PM, DeRuggerio K, Hoffmann B. Seventy-twohour returns may not be a good indicator of safety in the emergency department: a national study. Acad Emerg Med 2011; 18:390-7.

11. Pierce JM, Kellerman AL, Oster C. "Bounces": an analysis of short-term return visits to a public hospital emergency department. Ann Emerg Med 1990; 19:752-7.

12. Goh SH, Masayu MM, Teo PS, Tham AH, Low BY. Unplanned returns to the accident and emergency department--why do they come back? Ann Acad Med Singapore 1996; 25:541-6.

13. Kuan WS, Mahadevan M. Emergency unscheduled returns: can we do better? Singapore Med J 2009; 50:1068-71

14. Shy BD, Shapiro JS, Shearer PL, et al. A conceptual framework for improved analyses of 72-hour return cases. Am J Emerg Med 2015; 33:104-7. 
15. Rising KL, Padrez KA, O'Brien M, et al. Return visits to the emergency department: the patient perspective. Ann Emerg Med 2015; 65:377-386.e3.

16. Keith KD, Bocka JJ, Kobernick MS, Krome RL, Ross MA. Emergency department revisits. Ann Emerg Med 1989; 18:964-8.

17. Lerman B, Kobernick MS. Return visits to the emergency department J Emerg Med 1987; 5:359-62.

18. Milbrett $P$, Halm M. Characteristics and predictors of frequent utilization of emergency services. J Emerg Nurs 2009; 35:191-8; quiz 273.
19. O'Loughlin K, Hacking KA, Simmons N, et al. Paediatric unplanned reattendance rate: A\&E clinical quality indicators. Arch Dis Child 2013; 98:211-3.

20. Hasan M. Readmission of patients to hospital: still ill defined and poorly understood. Int J Qual Health Care 2001; 13:177-9.

21. Sun Y, Heng BH, Tay SY, Tan KB. Unplanned 3-day re-attendance rate at Emergency Department (ED) and hospital's bed occupancy rate (BOR). Int J Emerg Med 2015; 8:82. 